\title{
The immunological basis of tumor therapy by targeted delivery of
} TNFa to tumor vessels

\author{
Enrica Balza ${ }^{1}$, Lorenzo Mortara ${ }^{2}$, Francesca Sassi ${ }^{1}$, Stefano Monteghirfo ${ }^{1}$, \\ Barbara Carnemolla ${ }^{1}$, Patrizia Castellani ${ }^{1}$, Dario Neri ${ }^{3}$, Roberto Accollo*3, \\ Luciano Zardi ${ }^{4}$ and Laura Borsi ${ }^{1}$
}

\begin{abstract}
Address: ${ }^{1}$ Department of Translational Oncology, Istituto Nazionale per la Ricerca sul Cancro, Genoa, Italy, ${ }^{2}$ Department of Clinical and Biological Sciences, School of Medicine, University of Insubria, Varese, Italy, ${ }^{3}$ Department of Chemistry and Applied Biosciences, Institute of Pharmaceutical Sciences, Swiss Federal Institute of Technology, Zurich, Switzerland and ${ }^{4}$ Unit of Innovative Therapies, Istituto Giannina Gaslini, Centro Biotecnologie Avanzate, Genoa, Italy

* Corresponding author
\end{abstract}

from 2006 International Meeting of The Institute of Human Virology

Baltimore, USA. 17-2I November, 2006

Published: 21 December 2006

Retrovirology 2006, 3(SuppI I):PI doi:I0.I I86/1742-4690-3-SI-PI

(c) 2006 Balza et al; licensee BioMed Central Ltd.

L19mTNFa is a fusion protein constituted by the scFv L19 specific for the oncofetal ED-B domain of fibronectin and TNFa. Treatment with L19mTNFa, in combination with melphalan, induced complete tumor regression in $83 \%$ of BALB/c mice with WEHI-164 fibrosarcoma and 33\% of animals with C51 colon carcinoma. All cured mice rejected challenges with the same tumor cells and, in a very high percentage of animals, also challenges with syngeneic tumor cells of different histological origin. In adoptive immunity transfer experiments the splenocytes from C51-cured mice protected $100 \%$ of naive mice both from C51 colon carcinoma and from WEHI-164 fibrosarcoma. The splenocytes from WEHI-164-cured mice protected $100 \%$ of mice from the fibrosarcoma and $80 \%$ from the C51 colon carcinoma. Similar results were also obtained in adoptive immunity transfer experiments using severely immunodepressed SCID mice. Experiments using depleted splenocytes showed that $\mathrm{T}$ cells play a major role in tumor rejection. These data demonstrate that the selective targeting of mTNFa to the tumor enhances its immunostimulatory properties to the point of generating a therapeutic immune response against different histologically unrelated syngeneic tumors. These findings predicate treatment approaches for cancer patients based on the targeted delivery of TNFa to tumor vasculature. 